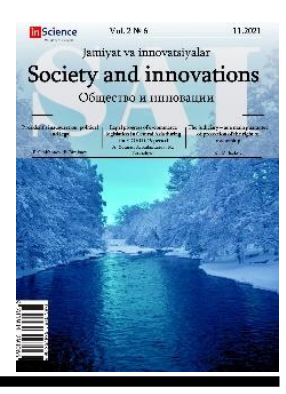

\title{
The importance of using clinical audit in teaching the subject of hematology
}

\author{
Lutfulla MAKHMONOV ${ }^{1}$, Feruza MAMATKULOVA2 \\ Kattabek ABDIEV ${ }^{3}$, Dilafruz AMEROVA ${ }^{4}$
}

Samarkand Medical Institute

\begin{tabular}{l} 
ARTICLE INFO \\
\hline Article history: \\
Received October 2021 \\
Received in revised form \\
15 October 2021 \\
Accepted 15 November 2021 \\
Available online \\
25 December 2021
\end{tabular}

Keywords:

clinical audit,

laboratory audit,

clinical audit project,

target design,

target domain,

target subdomain.

\begin{abstract}
This article deals with analysis the strengths and weaknesses of clinical audit, what is clinical audit, and how it is used. This systematic basis for validation can be adopted for all levels of assessment in medical education, from the individual assessment method to the approval of the assessment program as a whole.

The article also provides theoretical insights into the role of the use of clinical audit in the teaching of hematology.

2181-1415/C 2021 in Science LLC.

DOI: https://doi.org/10.47689/2181-1415-vol2-iss11/S-pp215-221

This is an open access article under the Attribution 4.0 International (CC BY 4.0) license (https://creativecommons.org/licenses/by/4.0/deed.ru)
\end{abstract}

\section{Gematologiya fanini o'qitishda klinik auditdan foydalanishning ahamiyati}

\section{Kalit so'zlar: \\ klinik audit, \\ laboratoriya auditi, \\ klinik audit loyihasi, \\ maqsadli dizayn, \\ maqsadli domen, \\ maqsadli subdomen.}

\begin{abstract}
ANNOTATSIYA
Ushbu maqolada klinik auditning kuchli va zaif tomonlari, klinik audit nima va undan qanday foydalanilishi tahlil qilinadi. Tasdiqlashning ushbu tizimli asosi tibbiy ta'limdagi baholashning barcha darajalari uchun, individual baholash usulidan tortib, umuman, baholash dasturini tasdiqlashgacha qabul qilinishi mumkin.
\end{abstract}

Shuningdek, maqolada gematologiyani o'qitishda klinik auditdan foydalanishning roli haqida nazariy qarashlar keltirilgan.

\footnotetext{
${ }^{1}$ Head of the Department of Hematology, Samarkand Medical Institute. Samarkand, Uzbekistan.

2 Assistant of the Department of Hematology, Samarkand Medical Institute. Samarkand, Uzbekistan.

${ }^{3}$ Associate Professor of the Department of Hematology, Samarkand Medical Institute. Samarkand, Uzbekistan.

${ }^{4}$ Assistant of the Department of Hematology, Samarkand Medical Institute. Samarkand, Uzbekistan.
} 


\section{Значение применения клинического аудита в процессе преподавания предмета гематология}

\author{
Ключевые слова: \\ клинический аудит, \\ лабораторный аудит, \\ проект клинического \\ аудита, целевая \\ конструкция, \\ целевой домен, \\ целевой субдомен.
}

\section{АННОТАЦИЯ}

В этой статье анализируются сильные и слабые стороны клинического аудита, что такое клинический аудит и как его использовать. Эта систематическая основа для валидации может быть принята для всех уровней оценки в медицинском образовании, от метода индивидуальной оценки до утверждения программы оценки в целом.

В статье также дается теоретическое представление о роли использования клинического аудита в преподавании гематологии.

Клинический аудит - это циклический и систематический обзор процессов, практик и результатов оказания медицинских услуг в соответствии с четко определенными критериями, основанными на фактических данных [6]. Систематические обзоры, как таковые важны в клинической практике, поскольку они делают упор на улучшение качества в стремлении соответствовать стандартам и возможности предоставлять пациентам передовую медицинскую помощь. При правильном исполнении, они обеспечивают прочную основу для объективного, систематического и постоянного улучшения ухода за пациентами.

Аудит, определяется как процесс улучшения качества, который направлен на возможность, улучшение ухода за пациентами и его результатов посредством систематического обзора помощи в соответствии с явными критериями и внедрения изменений [12]. Это часть процесса постоянного улучшения качества и ключевой элемент управления клинической практикой. Лабораторные аудиты оценивают компоненты лабораторных услуг; обеспечение обратной связи с сотрудниками и пользователями о функциях и эффективности лаборатории. Лабораторный аудит состоит из трех компонентов, а именно: преаналитическая фаза, аналитическая фаза и постаналитическая фаза [5]. Из-за цикла лабораторного качества надежность не может быть достигнута в клинической лаборатории только за счет контроля точности в одном компоненте процесса тестирования. Сертификация должна проводиться для всех функций лаборатории, но не для одного аналитического процесса [14]. В широкий круг преаналитических этапов входит следующее: запрос теста, сбор образцов, обработка и обработка перед полным распределением тестовых образцов на несколько рабочих станций [5; 12].

Исследования показывают, что ошибки только на преаналитической фазе могут составлять более 50\% ошибок в цикле тестирования [3]. В исследовании, проведенном в Италии, 6,6\% из 189 ошибок преаналитической фазы и постаналитической фазы были связаны с несоответствующей терапией (например, несоответствующее переливание крови или несоответствующее использование гепарина), а еще в 19\% ошибки были связаны с несоответствующими дальнейшими исследованиями, что увеличивает стоимость ухода [11]. Очень мало исследований изучали частоту и влияние неполных форм лабораторных запросов. Лабораторные ошибки имеют первостепенное значение, поскольку лабораторные данные влияют 
на 70\% медицинских диагнозов и могут существенно повлиять на успех и стоимость лечения пациента. По этим причинам предыдущие попытки уменьшить количество ошибок на аналитической фазе были оставлены [2], поскольку в настоящее время пре - и постаналитические процессы в лаборатории более уязвимы для ошибок [10], а иногда и предварительные. -аналитический учет фаз до 68\% лабораторных ошибок. Этот этап включает процедуры, которые не контролируются лабораторным персоналом и в основном выполняются вне лаборатории. Процедуры включают заполнение форм лабораторных запросов, идентификацию образцов, флеботомию, обработку образцов и транспортировку в лаборатории [13]. Бланки лабораторных запросов содержат информацию о запрошенном лабораторном тесте и демографические данные, такие как имя (имена), дату рождения, адрес, возраст и пол субъекта. Другие данные включают палату, номер лаборатории, имя врача, подпись врача, номер телефона или факса врача; клинические данные, статус пациента натощак и дата запроса.

Учебная программа по народонаселению и профилактическому здоровью в программе бакалавриата медицинского образования (MBBS) в Медицинской школе Университета Нотр-Дам в Австралии (UNDA) завершается на последнем году обучения, когда студенты выполняют контролируемый завершающий проект, известный как «проект клинического аудита». (САР), который составляет 10\% от их итоговой оценки [8]. Студенты должны определить SMART (конкретный, измеримый, достижимый, связанный и привязанный ко времени) стандарт, относящийся к оказанию медицинской помощи, который публикуется, принимается в качестве политики в области здравоохранения или широко принятый. После этого проводится критическая оценка доказательств, подтверждающих выбранный стандарт, прежде чем оценивать, соответствует ли оказываемая медицинская помощь этому стандарту. Студенты идентифицируют и консультируются с ключевыми заинтересованными сторонами, которые обычно являются персоналом, участвующим в предоставлении проверяемой помощи, на этапах планирования и распространения своей проверки. В соответствии с обычными процедурами в клинических условиях студенты должны получить письменное разрешение от соответствующего комитета по клиническому качеству и безопасности (или эквивалентного) службы здравоохранения, в которой они планируют проводить аудит. Чтобы соответствовать академическим требованиям Школы, студенты представляют предложение клинического аудита для формативной оценки их сверстниками и экзаменаторами (оценка для обучения) и заключительный отчет аудита для итоговой оценки (оценка обучения) в формате для публикации в медицинском журнале. Валидность, возможно, является наиболее важным фактором при оценке, поскольку она обеспечивает подтверждение того, что оценочные баллы интерпретируются и используются надлежащим образом и осмысленно. Современные теоретики, такие как Сэмюэл Мессик, Терри Дж. Крукс и его коллеги и Майкл Т. Кейн, рассматривают валидность как единую или единую концепцию [7; 9]. Этот современный взгляд на валидность был принят в качестве основы для пересмотренных Стандартов педагогического и психологического тестирования $[1,7]$. Вместо того, чтобы рассматриваться как просто характеристика инструмента оценки или теста, валидность больше связана со степенью, в которой уместность, значимость и полезность оценочных баллов допускают правильную интерпретацию. Таким образом, тест или инструмент оценки не следует 
рассматривать просто как действительный или недействительный. Валидность становится очевидной, и ее необходимо учитывать, когда оценки интерпретируются и используются для измерения успеваемости учащегося и определения необходимых действий. При этом уместность, значимость и полезность интерпретации и использования оценочных баллов неизбежно связаны с более фундаментальным вопросом, в первую очередь, соответствует ли инструмент оценки своей цели. Фактически, процесс подтверждения действительности является (или, по крайней мере, должен быть) циклическим процессом, который следует за жизненным циклом этапов проектирования, разработки, внедрения и оценки каждой оценки.

По словам Сэмюэля Мессика, существуют две основные всеобъемлющие категории угроз действительности оценок, а именно построение нерелевантной дисперсии (CIV) и построение с учетом представления (CUR) [9, 8]. CIV представляет собой угрозу достоверности из-за неконтролируемых посторонних переменных, которые могут влияет на успеваемость учащихся при оценивании и, следовательно, влияет на точность результатов и законность последующих решений, принимаемых на основе этих результатов. CUR, с другой стороны, возникает, когда то, что оценивается, не отражает соответствующих знаний, навыков или отношения, что ставит под угрозу осмысленную и надлежащую интерпретацию и использование полученных результатов.

Недавний призыв к единому взгляду на валидность является императивным и своевременным, поскольку он подчеркивает тот факт, что эта конструкция существует в континууме, в отличие от общепринятого бинарного понятия «действительный» или «недействительный» [7; 9]. Часто практика оценки сосредоточена исключительно на надежности и упускает из виду более сложный вопрос достоверности, который, в отличие от надежности, не может быть измерен численно. Вместо этого проверка достоверности оценки включает в себя процесс расследования и сбора данных для выявления свидетельств, необходимых для опровержения значительных угроз действительности на каждом этапе цикла оценки. Таким образом, современные теоретики подчеркивают важность исследования по валидации оценок, и что к валидности при интерпретации и использовании оценочных баллов следует подходить как гипотезу, а не предполагать.

Это валидационное исследование является ответом на вышеупомянутый призыв к целостному и единому взгляду на валидацию и оценочные исследования. Это также одна из многих параллельных инициатив со стороны медицинской школы ЮНДА по постоянному совершенствованию и уточнению каждого компонента оценки - согласованные усилия по созданию более надежной, значимой и справедливой программы оценки.

Цель проекта клинического аудита

Проект клинического аудита - это краеугольный камень, позволяющий студентам синтезировать то, что они узнали от населения, и учебную программу по профилактическому здоровью (включая доказательную медицину, исследования, системы здравоохранения, качество и безопасность, а также профессионализм) в течение первых трех лет и применять его в реальное клиническое рабочее место для измерения и пропаганды улучшения качества в каком-либо аспекте ухода за пациентами. 
Интерпретация и использование партитуры

- Результаты проекта клинического аудита составляют $10 \%$ от общей оценки студента за последний год

- Баллы проекта клинического аудита используются для выявления студентов, которые продемонстрировали значительный недостаток в своей способности проводить и сообщать о проекте клинического аудита, чтобы эти студенты могли пройти программу исправления, либо для улучшения существующего проекта аудита, либо для выполнения нового проекта.

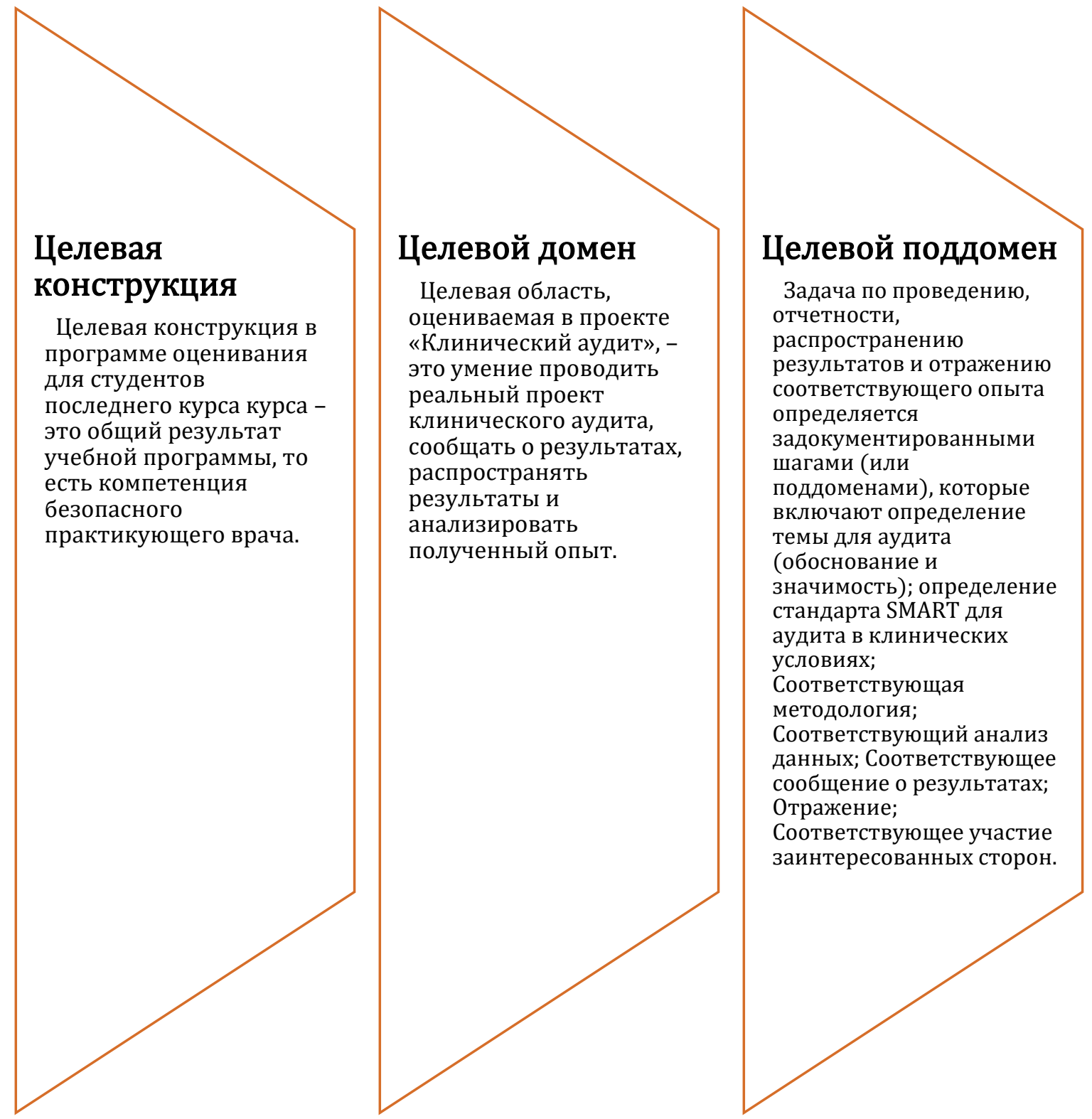

Аргумент интерпретации, изложенный выше, хотя и определен в соответствии с соответствующими составными частями, все же был относительно широкими утверждениями, которые требовали дальнейшей распаковки для проверки действительности на более детальном уровне. Таким образом, цепная модель образовательной оценки Крукса, Кейна и Коэна [4] использовалась для постановки конкретного вопроса проверки на каждом этапе проекта клинического аудита: 
Вопрос проверки 1. Соответствует ли проект клинического аудита своей цели?

Вопрос проверки 2. Являются ли методологические и когнитивные процессы, задействованные в проекте клинического аудита, актуальными (связь между проектированием и планированием)?

Вопрос проверки 3. Действительно ли суждения экзаменаторов об успеваемости студента отражают его способности в оцениваемой области?

Вопрос проверки 4. Можно ли обобщить баллы клинического аудита на все возможные баллы по темам клинического аудита и ко всем экзаменаторам, оценивающим отчеты?

Вопрос проверки 5. Оценивают ли совокупные итоговые баллы отчетов о клиническом аудите одну общую основную конструкцию? Есть ли возможности построения непредставлении?

Вопрос валидации 6. Имеются ли достаточные концептуальные и эмпирические данные в поддержку экстраполяции из целевой области, оцененной в рамках проекта клинического аудита, на целевую конструкцию, то есть общую компетентность безопасного выпускника медицинского факультета?

Вопрос проверки 7. Основана ли оценка баллов на достаточной информации об оценке, включая ограничения, возникающие из-за ошибок измерения?

Вопрос проверки 8. Существует ли четкое, справедливое, явное и хорошо изложенное правило принятия решения в качестве основы для важных решений, основанных на оценках клинического аудита?

Вопрос проверки 9. Каковы образовательные возможности и влияние проекта клинического аудита? Есть ли доказательства непредвиденных последствий? Каковы социальные последствия выполнения студентами проекта клинического аудита?

Одним из важных выводов цепной модели оценивания образования Крукса, Кейна и Коэна является то, что во всех методах оценивания есть ряд взаимосвязанных этапов, и угрозы валидности могут возникать из-за практик на каждом звене оценочного цикла. Если какое-либо из этих звеньев является слабым или действительно разорванным, это влияет на общую целостность оценочных баллов. Таким образом, крайне важно, чтобы разработчики оценки выявляли практики с вероятными угрозами действительности, то есть самое слабое звено, чтобы можно было попытаться создать механизм обеспечения качества для смягчения этих угроз и укрепления общей целостности.

\section{БИБЛИОГРАФИЧЕСКИЕ ССЫЛКИ:}

1. American Educational Research Association, American Psychological Association, National Council on Measurement in Education, Joint Committee on Standards for Educational, Psychological Testing (US). Standards for educational and psychological testing. Washington, DC: American Educational Research Association; 1999.

2. Bonini P, Plebani M, Ceriotti F, Rubboli F. Errors in laboratory medicine. Clinical Chemistry. 2002; 48:691-698. [PubMed] [Google Scholar].

3. Boone D.J., Steindel S.J., Herron R. Transfusion medicine monitoring practices: a study of the College of American Pathologists / Centers for Disease Control and Prevention Outcomes Working Group. Archives of Pathology \& Laboratory Medicine. 1995; 119:9991006. [PubMed] [Google Scholar]. 
4. Crooks T.J., Kane M.T., Cohen A.S. Threats to the valid use of assessments. Assessment in Education: Principles, Policy and Practice. 1996; 3(3):265-86.

5. Erasmus R.T., Zemlin A.E. Clinical audit in the laboratory. Journal of Clinical Pathology. 2009; 62:593-597. [PubMed] [Google Scholar].

6. Esposito P, Dal Canton A. Clinical audit, a valuable tool to improve quality of care: General methodology and applications in nephrology. World J Nephrol. 2014;3:249-255. doi: 10.5527/wjn.v3.i4.249. [PMC free article] [PubMed] [CrossRef] [Google Scholar]

7. Kane MT. Validating the Interpretations and Uses of Test Scores. Journal of Educational Measurement. 2013; 50:1-73. doi: 10.1111 / jedm.12000. [CrossRef] [Google Scholar]

8. Mak D.B., Miflin B. Clinical audit in the final year of undergraduate medical education: towards better care of future generations. Med Teach. 2012; 34:251-257. DOI: 10.3109/0142159X.2012.660216. [PubMed] [CrossRef] [Google Scholar].

9. Messick S. Meaning and Values in Test Validation: The Science and Ethics of Assessment. Educational Researcher. 1989; 18:5-11. doi: 10.2307/1175249. [CrossRef] [Google Scholar].

10. Plebani M., Carraro P. Mistakes in a stat laboratory: types and frequency. Clinical Chemistry. 1997; 43:1348-1351. [PubMed] [Google Scholar].

11. Plebani M. Errors in clinical laboratories or errors in laboratory medicine? Clinical Chemistry and Laboratory Medicine. 2006; 44:750-759. [PubMed] [Google Scholar].

12. Scrivener R., Morrell C., Baker R., Redsell S., Shaw E., Stevenson K., Pink D., Bromwich N. Principles for best practice in clinical audit. International Journal for Quality in Health Care. 2002; 15:87-97. [Google Scholar].

13. Vanker N., Wyk J.V., Zemlin A.E., Erasmus R.T. A Six sigma approach to the rate and clinical effect of registration errors in a laboratory. Journal of Clinical Pathology. 2010; 63:434-437. [PubMed] [Google Scholar].

14. Wiwanitkit $\mathrm{V}$. Types and frequency of preanalytical mistakes in the first Thai ISO 9002:1994 certified clinical laboratory, a 6 - month monitoring. BMC Clinical Pathology. 2001; 1:5. [PMC free article] [PubMed] [Google Scholar]. 